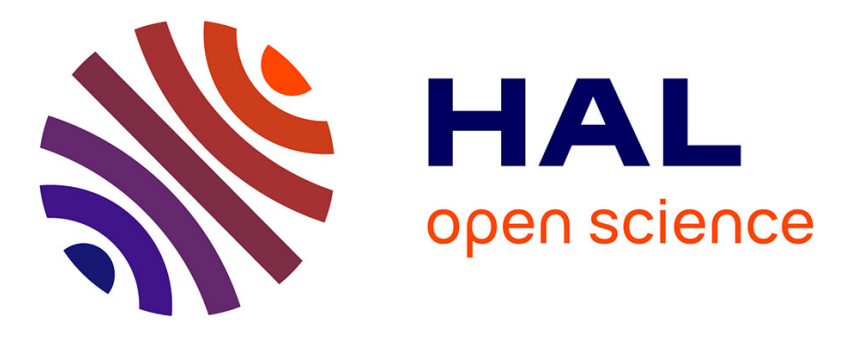

\title{
Integrated microfluidic device for the separation, decomposition and detection of low molecular weight S-nitrosothiols
}

Gerson Duarte-Junior, Abdulghani Ismail, Sophie Griveau, Fanny d'Orlyé, José Alberto Fracassi da Silva, Wendell Coltro, Fethi Bedioui, Anne Varenne

\section{To cite this version:}

Gerson Duarte-Junior, Abdulghani Ismail, Sophie Griveau, Fanny d'Orlyé, José Alberto Fracassi da Silva, et al.. Integrated microfluidic device for the separation, decomposition and detection of low molecular weight S-nitrosothiols. Analyst, 2019, 144 (1), pp.180-185. 10.1039/c8an00757h . hal02159778

\section{HAL Id: hal-02159778 \\ https://hal.science/hal-02159778}

Submitted on 19 Jun 2019

HAL is a multi-disciplinary open access archive for the deposit and dissemination of scientific research documents, whether they are published or not. The documents may come from teaching and research institutions in France or abroad, or from public or private research centers.
L'archive ouverte pluridisciplinaire HAL, est destinée au dépôt et à la diffusion de documents scientifiques de niveau recherche, publiés ou non, émanant des établissements d'enseignement et de recherche français ou étrangers, des laboratoires publics ou privés. 


\section{Integrated microfluidic device for the separation, decomposition and detection of low molecular weight S-nitrosothiols}

5 Gerson F. Duarte-Junior ${ }^{\mathrm{a}, \mathrm{b}}$, Abdulghani Ismail ${ }^{\mathrm{a}}$, Sophie Griveau ${ }^{\mathrm{a}}$, Fanny

6 d'Orlyé $^{a}$, José Alberto Fracassi da Silva ${ }^{\mathrm{e}}$, Wendell K. T. Coltro ${ }^{\mathrm{b}}$, Fethi

7 Bedioui ${ }^{a}$, Anne Varenne ${ }^{\mathrm{a}^{*}}$

$9{ }^{a}$ Chimie ParisTech, PSL Research University, INSERM 1022, CNRS 8258 ,

10 Paris Descartes, Unité de Technologies Chimiques et Biologiques pour la

11 Santé, 75005 Paris, France

12 b Instituto de Química, Universidade Federal de Goiás, Campus

13 Samambaia, Goiânia, GO, 74690-900, Brazil

14 ' Instituto de Química, Universidade Estadual de Campinas, UNICAMP,

15 Campinas, SP, 13083-970, Brazil

$17{ }^{*}$ Corresponding Author. (anne.varenne@chimieparistech.psl.eu) 


\section{Abstract}

19 S-nitrosothiols (RSNOs) are considered as biological circulating stocks of

20 nitric oxide (NO) that have many roles in-vivo. The variation of RSNOs

21 proportion occurs in several diseases, which makes them potent

22 biomarkers. The identification and quantitation of each RSNO is therefore

23 important for biomedical studies. For now, miniaturized devices have been

24 used to detect RSNOs, based on their total quantitation without a preceding

25 separation step. This study reports on an original and integrated

26 microdevice allowing for the successive separation of low molecular weight

27 RSNOs, their decomposition under metal catalysis, and the quantitation by

28 amperometric detection of the produced nitrite, leading to their quantitation

29 in a single run. For this purpose, a commercial SU-8/Pyrex microfluidic

30 system was coupled to a portable and wireless potentiostat. Different

31 operating and running parameters were optimized to achieve the best

32 analytical performance allowing for LODs of $20 \mu \mathrm{M}$. The simultaneous

33 separation of S-nitrosoglutathione and S-nitrosocysteine was successfully

34 obtained within $75 \mathrm{~s}$. 


\section{Introduction}

38 S-Nitrosothiols (RSNOs) are nitric oxide (NO) carrier molecules that play

39 important roles in several physiological functions (vasodilatation and

40 relaxation $^{1,2}$, antiplatelet aggregation ${ }^{3,}{ }^{4}$, antimicrobial ${ }^{5}$, regulation and

41 signaling protein function ${ }^{6} \ldots$ ) and pathological events (neurodegenerative

42 diseases such as Parkinson and Alzheimer ${ }^{7}$, apoptosis ${ }^{8}$, chronic obstructive

43 pulmonary disease ${ }^{9}$, preeclampsia ${ }^{10}$, diabetes $\left.{ }^{11} \ldots\right)$. RSNOs can be divided

44 into low molecular weight (LMW) and high molecular weight (HMW)

45 RSNOs. Although there is no defined border in terms of molecular mass, it

46 is common to use the term "low molecular weight" for peptides and

47 aminoacid S-nitrosothiols (such as S-nitrosoglutathione (GSNO) and S-

48 nitrosocysteine (CySNO)) and "high molecular weight" for s-nitrosylated

49 proteins (such as S-nitrosoalbumin (AlbSNO) and S-nitrosohemoglobin

50 (HbSNO)). RSNOs store, transport and release NO. They can also inter-

51 exchange NO through transnitrosation reaction ${ }^{13}$. The variation of RSNOs

52 concentration has been shown to occur in many diseases ${ }^{12}$. For all these

53 reasons, the development of powerful methodologies for the simultaneous

54 quantitation of all RSNOs in a biological sample is crucial.

55 Numerous methods have been developed for RSNOs analysis, based on

56 direct or indirect detection. Examples of direct detection consist in the 
57 separation of RSNOs from other species by capillary electrophoresis (CE)

58 or liquid chromatography followed by mass spectrometric or

59 spectrophotometric detection ${ }^{14}$. For example, the simultaneous separation

60 of S-nitrosoglutathione (GSNO) and S-nitrosocysteine (CySNO) was

61 performed by our group using conventional CE equipped with capacitively

62 coupled contactless conductivity detection but in a conventional system ${ }^{15}$.

63 In another work, the simultaneous separation of GSNO, GSH, glutathione

64 sulfonic and sulfinic acid by CE coupled to mass spectrometry was

65 obtained $^{14}$. Most standard methods developed for RSNOs quantitation

66 reported in the literature remain indirect. They are based on the detection of

67 their decomposition products ${ }^{16}$, through homolytic or heterolytic cleavage of

68 S-NO bond generating $\mathrm{NO}$ or $\mathrm{NO}^{+}$leading finally to $\mathrm{NO}_{2}^{-}$. These

69 decomposition products are then detected by spectrophotometry,

70 fluorimetry, electrochemistry or chemiluminescence ${ }^{16,17}$. Various reagents

71 have been used to decompose RSNOs, such as metal cations ${ }^{18} \mathrm{(Hg}^{2+}$,

$72 \mathrm{Cu}^{+}$), light ${ }^{19}$ and heat $^{20}$, leading to different decomposition products: $\mathrm{NO}$ is

73 generated $^{18}$ if $\mathrm{Cu}^{+}$, light or heat are employed, whereas nitrite is directly

74 generated ${ }^{18,21}$ when $\mathrm{Hg}^{2+}$ or $\mathrm{Ag}^{+}$are used.

75 Nowadays, miniaturization in chemical analysis has become a powerful tool

76 contributing to reduce the samples/reagents amount, analysis time and 
77 waste generation. Such an approach can be beneficial for the quantitation

78 of RSNOs. Indeed, our group has recently reported on the colorimetric

79 analysis of RSNOs in a microfluidic paper-based anaytical device ${ }^{22}$. This

80 system allowed to perform analysis of total RSNOs in plasma samples

81 without any separation step. Other approaches were proposed by Hunter et

82 al. for $\mathrm{NO}^{23}$ and total RSNO detection ${ }^{24}$ (after light decomposition) using a

83 single PDMS microfluidic channel with amperometric detection. In all cases

84 no separation of RSNOs occurred before detection in these miniaturized

85 devices. Also, Gunasekara et $\mathrm{al}^{25}$ used microchip capillary electrophoresis

86 (MCE) with amperometric detection to separate a NO donor

87 (DEA-NONOate or Proli-NONOate) from NO and nitrite in less than one 88 minute. Tu et al. ${ }^{26}$ used MCE with fluorescence detection to separate and 89 detect NO, reduced glutathione (GSH) and cysteine (Cys). Herein we report

90 the design and optimization of a single-run MCE analytical strategy allowing

91 for the first time the simultaneous quantitation of two low molecular weight

92 RSNOs (S-nitrosoglutathione and S-nitrosocysteine), thanks to the

93 integration of successive electrokinetic separation, RSNO decomposition by

$94 \mathrm{Hg}^{2+}$ to nitrite and nitrite quantitation by amperometry. 


\section{Experimental}

96 Chemicals

97 All reagents were of analytical grade and used as received. L-arginine 98 (ARG), 2-(N-morpholino) ethanesulfonic acid (MES), acetic acid (HAc), L99 histidine (His), sodium tetraborate, Sodium Nitrite, N-acetyl-p-aminophenol 100 (Paracetamol), Mercury(II) Chloride, EDTA, hydrochloric acid, sodium 101 phosphate monobasic, sodium phosphate dibasic, L-cysteine (Cys) and 102 reduced glutathione (GSH), were purchased from Sigma Aldrich (St. Louis, $103 \mathrm{MO}$, USA). All aqueous solutions were made using ultra-pure water with a 104 resistivity of $18.2 \mathrm{M} \Omega . \mathrm{cm}$ from a Pure Lab Flex system (ELGA Labwater, 105 France).

106 Synthesis of S-nitrosothiols

107 GSNO was synthesized as described elsewhere ${ }^{27}$. Briefly, an equimolar 108 amount of nitrite was added to the equimolar amount of $\mathrm{GSH}$ and $\mathrm{HCl}$. The 109 resulting pure solid was rinsed once with $80 \%$ acetone, twice with $100 \%$ 110 acetone and three times with diethyl ether and then stocked in the dark at $11120^{\circ} \mathrm{C}$.

112 S-Nitrosocysteine (CySNO) was daily synthesized using the method 113 described by Peterson and coworkers ${ }^{28}$. Briefly, solutions of $5 \mathrm{mM}$ CysNO 
114 were prepared by reacting cysteine with an equimolar concentration of 115 nitrite in acidic medium $(0.1 \mathrm{M} \mathrm{HCl})$ in a dark flask to avoid light

116 decomposition. After 5 min, more than $90 \%$ of cysteine was converted into

117 CysNO. The solution was neutralized by 0.1 M PBS buffer $(\mathrm{pH} 7.4)$ 118 containing $0.5 \mathrm{mM}$ EDTA to prevent decomposition by trace metal cation 119 contaminants.

120 Final concentrations of RSNOs were determined spectrophotometrically in 121 aqueous solution at $335 \mathrm{~nm}\left(\varepsilon=586\right.$ and $503 \mathrm{M}^{-1} \mathrm{~cm}^{-1}$ for GSNO and 122 CysNO, respectively $)^{29}$.

123 Instrumentation

124 Electrophoretic experiments were performed using a SU-8/Pyrex 125 microchips with integrated micro band platinum electrodes at the outlet end 126 of the separation channel from Micrux Technologies (Oviedo, Spain) (MCE127 SU8-Pt001T) (Figure 1). Only working (WE) and reference (RE) electrodes 128 were used, with widths of $50 \mu \mathrm{m}$ and $250 \mu \mathrm{m}$, respectively. The separation 129 and injection channel length were $35 \mathrm{~mm}$ and $10 \mathrm{~mm}$, respectively. The 130 microchannels width and depth were $50 \mu \mathrm{m}$ and $20 \mu \mathrm{m}$, respectively. A 131 microfluidic platform (Oviedo, Spain) (MCE-HOLDER-DC02) was used to 132 interface the microchip with the amperometric detector and the high voltage 
133 source. The high voltage source was a programmable HVS448-3000V 8-

134 channels high-voltage supply (LabSmith Inc., CA, USA) controlled by

135 Sequence software v.1.165. Amperometric detection was performed by a

136 modified model 9051, 2-channel, wireless, portable and electrically isolated

137 potentiostat (Pinnacle Technology, Lawrence, KS. USA) operating in a

138 two-electrode format at a $5 \mathrm{~Hz}$ sampling rate (gain=5 $000000 \mathrm{~V} / \mathrm{A}$, 139 resolution $=30 \mathrm{fA}$ ). This potentiostat is isolated which eliminates 140 interferences from the high voltage power supply system used for the 141 separation. This potentiostat was controlled by Sirenia Acquisition Software 142 v.1.7.6. The WE and RE were connected to the corresponding electrodes 143 using the commercial chip holder.
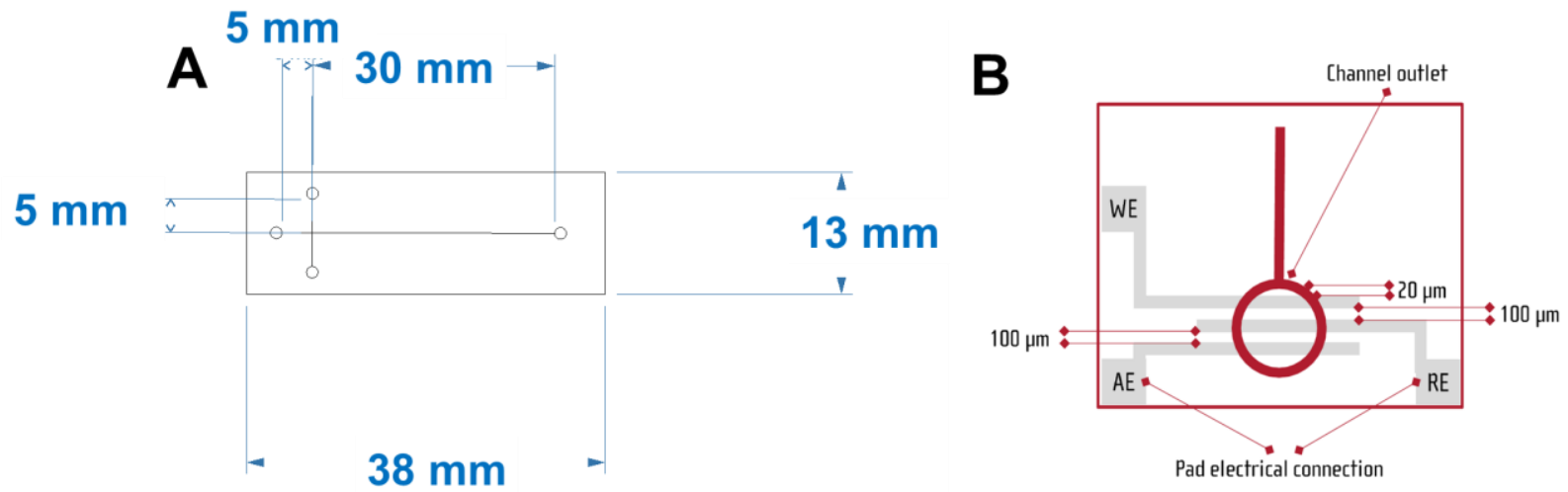

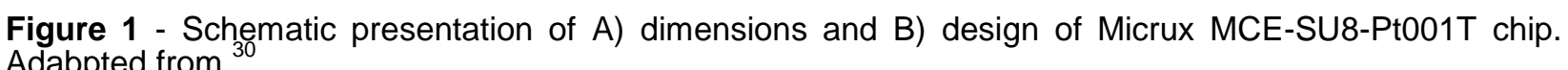

$148 \quad C^{4} D$ detection was performed using a commercial detector model ER815 149 acquired from eDAQ Pty (Denistone East, Australia). A microfluidic 
150 platform EDAQ ET121 containing external electrodes was used to interface

151 a commercial PMMA microchip (model 02-0750-0082-01, ChipShop, Jena,

152 Netherlands) with the detection system. This microchip layout comprised

153 separation and injection channels $(50 \mu \mathrm{m}$ wide/deep) with 87 and $10 \mathrm{~mm}$

154 long, respectively.

155 Electrophoresis and Decomposition procedure

156 Prior to analysis, microchannels were conditioned with $0.1 \mathrm{M} \mathrm{NaOH}$, 157 deionized water and running buffer. Samples of RSNOs and paracetamol

158 ( $1 \mathrm{mM}$ each) were electrokinetically injected by gated mode ${ }^{31}$ by applying 159 potentials of $800 \mathrm{~V}$ and $1000 \mathrm{~V}$ to sample and buffer reservoirs, 160 respectively, while both waste reservoirs were grounded for the loading 161 step. The injection was performed by floating the potential at the buffer 162 reservoir for $3 \mathrm{~s}$, giving the start of the separation step. The same 163 procedure but under reversed polarity was performed for nitrite $(1 \mathrm{mM})$ 164 quantitation. For the decomposition step, $\mathrm{HgCl}_{2}(10 \mathrm{mM})$ was added in the 165 detection reservoir. Before RSNOs reach the buffer waste reservoir the 166 polarity was inverted allowing detection of nitrite generated from 167 decomposition. For amperometric detection of nitrite and paracetamol, 168 potentials from 0.7 to $1.2 \mathrm{~V}$ vs. Pt were applied. Analysis using $C^{4} D$ 169 detection was realized under the same electrophoretic conditions and 
170 detection was performed applying a sinusidal signal of $600 \mathrm{kHz}$ and

$17190 \mathrm{~V}_{\text {peak-to-peak. }}$ 


\section{Results and discussion}

173 Herein a method for successive separation, decomposition, and detection

174 of RSNOs is proposed by integrating all the three steps in a microfluidic

175 device. In order to achieve this goal, the decomposition should be (i) much

176 faster than the separation process (otherwise the peaks originating from

177 different RSNOs will overlap) (ii) quantitable and (iii) the decomposition

178 product should be stable through the analysis time and operating

179 conditions. It is well known that the decomposition product of RSNOs

180 depends on the decomposition agent. As indicated above, the use of $\mathrm{Cu}^{+}$or

181 light leads to homolytic cleavage and to the formation of $\mathrm{NO}$, while the use

182 of $\mathrm{Hg}^{2+}$ leads to heterolytic cleavage and to the production of $\mathrm{NO}^{+}$that

183 transforms immediately to $\mathrm{NO}_{2}^{-}$. Decomposition of RSNOs by light is slow

184 (tenth of minutes are needed to decompose the sample ${ }^{15}$ ) and only partial

185 decomposition of RSNOs have be obtained by Hunter et $\mathrm{al}^{24}$ in a

186 microfluidic device using $530 \mathrm{~nm}$ LED. $\mathrm{Cu}^{+}$decomposition is faster than

187 light decomposition but still insufficient $\left(>2 \mathrm{~min}^{32}\right)$ in comparison with the

188 separation time scale. Moreover $\mathrm{Cu}^{+}$is poorly soluble and stable in aquous

189 solution and it is usually produced by reduction of $\mathrm{Cu}^{2+}$ with reducing agent

190 such as GSH. Decomposition using $\mathrm{Cu}^{2+}$ is affected by the variation of GSH

191 concentration in the sample, which is difficult to control ${ }^{32}$. Decomposition 
192 using mercuric (II) is instantaneous leading to $\mathrm{NO}_{2}^{-}$which is stable and 193 electroacive ${ }^{33}$. Consequently, $\mathrm{Hg}^{2+}$ was chosen as the decomposition 194 agent.

195 BGE plays an important role in the migration and electrochemical detection 196 steps in micro chip electrophoresis (MCE) ${ }^{34,35}$. As the objective was the 197 detection of nitrite generated from RSNO decomposition, the BGE 198 optimization was focused on nitrite signal/noise $(\mathrm{S} / \mathrm{N})$ ratio during detection. 199 Several BGEs usually used for biological samples during MCE were tested 200 : $20 \mathrm{mM} \mathrm{MES} \mathrm{/} \mathrm{His} \mathrm{(pH} \mathrm{6.0),} 20 \mathrm{mM}$ MES / Arg (pH 7.5), $20 \mathrm{mM} \mathrm{Arg} \mathrm{/}$ 201 Acetic Acid ( $\mathrm{pH} 5.8$ ). Nitrite (1 mM) was injected in the gated mode (see 202 experimental section), separated and detected by amperometry using these 203 various BGEs. For each BGE, the detection potential applied between WE 204 and RE was varied from $+0.5 \mathrm{~V}$ to $+1.5 \mathrm{~V}$ keeping constant same 205 electrophoretic injection and separation conditions. The optimal potential for 206 nitrite detection in all BGE was 0.7 V. Figure 2 shows electropherograms 207 for the separation of nittire in various BGE. $20 \mathrm{mM} \mathrm{MES/His}(\mathrm{pH} .6 .0)$ 208 provided the highest amperometric detection signal. However, it was not 209 selected for this design as $\mathrm{Hg}^{2+}$, that will be used as the decomposition 210 reagent for $\mathrm{RSNO}$, reacts with histidine to form a precipitate. Although 211 MES/Arg leads to the highest signal intensity, it however results in the 
212 lowest signal to noise ratio. Therefore, the selected BGE, leading to the

213 highest $\mathrm{S} / \mathrm{N}$ ratio without interference with other molecules in the solution, 214 was $20 \mathrm{mM}$ Arg adjusted to $\mathrm{pH} 5.8$ with HAc.

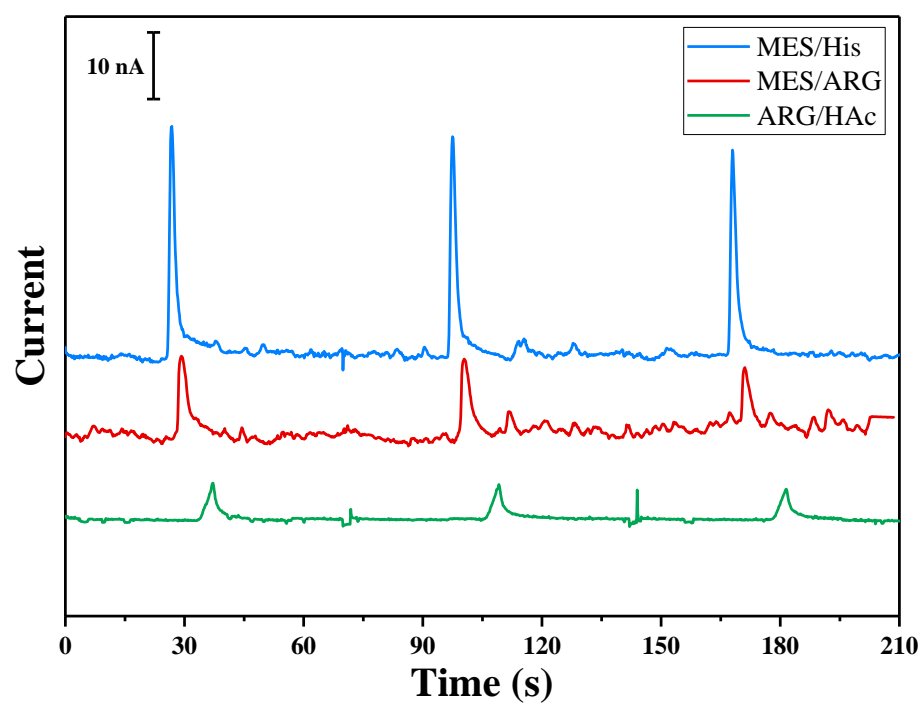

215

216 Figure 2 - Electrophoretic separation of $1 \mathrm{mM}$ nitrite in SU-8/Pyrex microchip. BGE: MES (20 mM)/His 217 (20 mM) pH 6.0 in blue, MES $(20 \mathrm{mM}) / \operatorname{Arg}(20 \mathrm{mM}) \mathrm{pH} 7.5$ in red, $\operatorname{Arg}(20 \mathrm{mM}) \mathrm{pH} 5.8$ adjusted with 218 Acetic Acid in green. Gated injection V1=-800V, V2 $=-1000 \mathrm{~V}$, injection time 3s, successive injections: 70s, 219 detection $0.7 \mathrm{~V}$ vs Pt.

221 For an optimized on-chip integration of the three steps involved in RSNOs 222 characterization (separation, decomposition and detection) in the microchip, 223 the apparent mobilities of each of the compounds (different RSNOs, nitrite, 224 mercury) were determined. The overall procedure was first optimized for 225 GSNO, as it is the most abundant low molecular weight RSNO. Control 226 experiments by direct addition of nitrite into the BW reservoir, with or 227 without application of electrophoretic voltage resulted in an amperometric 
228 signal, proving the efficiency of the electrochemical detection step. A 229 neutral electroactive marker (paracetamol) allowed determining the 230 electroosmotic mobility as $1.85 \pm 0.07 \times 10^{-4} \mathrm{~cm}^{2} \cdot V^{-1} \cdot \mathrm{s}^{-1}$ and GSNO 231 electrophoretic mobility was determined as $-0.64 \pm 0.06 \times 10^{-4} \mathrm{~cm}^{2} \cdot \mathrm{V}^{-1} \cdot \mathrm{s}^{-1}$, 232 employing a $C^{4} D$ detector, as GSNO is not electroactive (results not 233 shown).

234 Under these experimental conditions, GSNO migrates towards the detector 235 under a positive polarity. The device was primarily developed as follows: 236 GSNO was electrokinetically injected from the sample reservoir (S) in the 237 gated mode in positive polarity (see experimental section). $\mathrm{Hg}^{2+}$ was 238 introduced in the waste reservoir (connected to the cathode) where it 239 should decompose GSNO into nitrite upon reaching the buffer waste 240 reservoir (BW). However, no amperometric signal was observed (results not 241 shown). One hypothesis is based on the fact that $\mathrm{Hg}^{2+}$ undergoes diffusion 242 from the BW within the separation channel, inducing the GSNO 243 decomposition within the separation channel. As nitrite electrophoretic 244 mobility under these experimental conditions $\left(-4.25 \times 10^{-4} \mathrm{~cm}^{2} \cdot \mathrm{V}^{-1} \cdot \mathrm{s}^{-1}\right)$ is 245 higher in absolute value than the electroosmotic mobility, nitrite moves back 246 to the sample reservoir (S) instead of the BW reservoir where it should be 247 detected. 
A new design was then developed, including an additional step allowing for

249 voltage inversion just before GSNO decomposition (Figure 3A). In this new

250 design, the loading, injection and separation steps were performed under

251 positive polarity (step I, II and III, respectively), $\mathrm{Hg}^{2+}$ is added before GSNO

252 reaches the channel end in step III, and polarity is inverted (step IV). This

253 inversion of polarity leads to the migration of $\mathrm{Hg}^{2+}$ and GSNO to the sample

254 reservoir (S). As $\mathrm{Hg}^{2+}$ migrates faster than GSNO, the migration zone of

$255 \mathrm{Hg}^{2+}$ enters that of GSNO, allowing for GSNO decomposition. The

256 produced nitrite migrates towards the BW reservoir and is detected (see

257 detail in step IV, Figure 3A). A typical electropherogram obtained

258 characterizing all the analytical steps is presented in Figure. 3B. Control

259 experiments (without $\mathrm{Hg}^{2+}$ or without GSNO) did not show any signal (data

260 not shown).
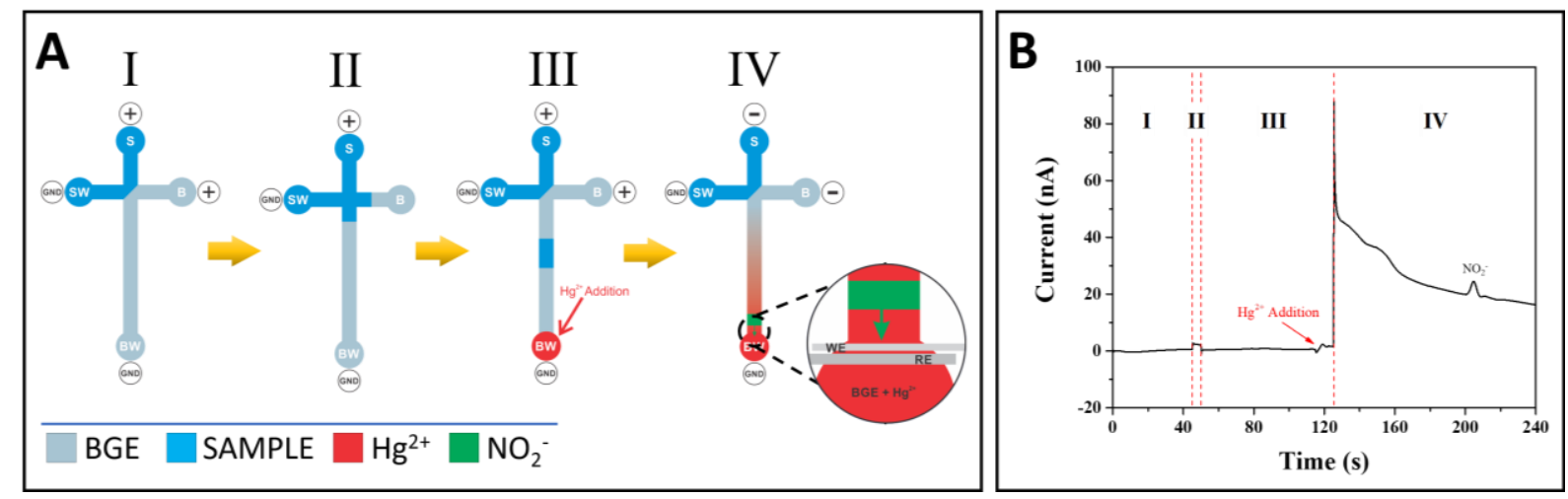

Figure 3 - (A) Scheme of the main steps for RSNOs quantitation. Loading step (I): Voltages of 800 and $1000 \mathrm{~V}$ are applied during $45 \mathrm{~s}$ to the sample (S) and buffer (B) reservoirs, respectively, grounding both waste reservoirs (SW and BW). Injection step (II): samples are injected into separation channel by floating the voltage applied to B reservoir during $3 \mathrm{~s}$. Migration step (III): The potentials were then reestablished to step I condition allowing the migration of RSNO sample towards separation channel. $15 \mathrm{~s}$ before the end of this step, $\mathrm{Hg}^{2+}$ was added to BW reservoir. Inversion and detection step (IV): In this 
step the potential polarity is inverted which leads to the migration of $\mathrm{Hg}^{2+}$ into the separation channel faster than RSNO leading to RSNO decomposition. This is followed by nascent nitrite (in green) opposite migration towards the electrodes and detection by applying a potential of $1.2 \mathrm{~V} v s \mathrm{Pt}$. (B) Typical electropherogram obtained for GSNO (1 mmol/L) analysis characterising all the steps of the process.

273 Therefore, the overall integrated protocol includes (1) a separation of the 274 RSNOs under positive polarity, (2) an inversion of the separation polarity,

275 (3) a decomposition of RSNO thanks to the on-line crossing and mixing of $276 \mathrm{RSNO}$ and $\mathrm{Hg}^{2+}$ zones, respectively, due to different migration velocities, (4) the migration of the produced nitrite to the detector. So as to further 278 optimize the experimental conditions to improve the limit of detection for 279 nitrite, three parameters were studied; the BGE ionic strength (from 10 to $28050 \mathrm{mM}$ ), the detection voltage (from 0.5 to $1.5 \mathrm{~V} v s P t$ ) and the time of 281 polarity inversion. Considering the two first parameters, best signal 282 intensities and $\mathrm{S} / \mathrm{N}$ ratios for nitrite detection were obtained for a detection 283 potential of $1.2 \mathrm{~V}$ (Figure 4). 


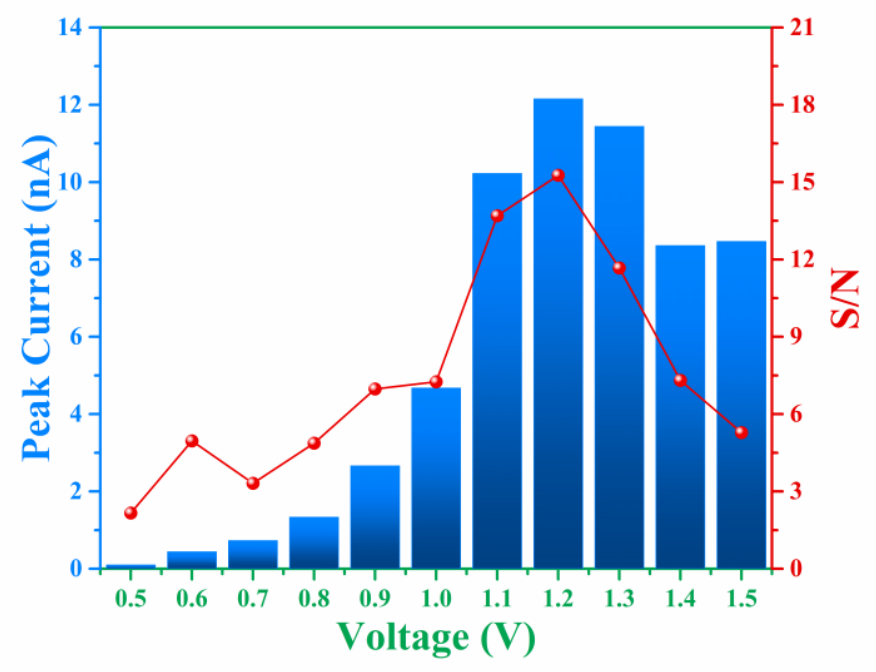

Figure 4 - Histogram of peak current (in $n A)$ and signal-to-noise ratio $(\mathrm{S} / \mathrm{N})$ of nitrite $(1 \mathrm{mM})$ injection in SU-8/Pyrex microchip obtained by variation of the detection potential from 0.5 to $1.5 \mathrm{~V}$ vs Pt. BGE: $20 \mathrm{mM}$ arginine solution adjusted at $\mathrm{pH} 5.6$ with acetic acid.

The time of polarity inversion is a crucial parameter as it should allow for 290 the best separation of the RSNOs, their total decomposition and their 291 optimal detection. For each studied compound, the electroosmotic and 292 apparent mobilities must therefore be determined. For RSNOs of positive 293 apparent mobility, the time of inversion should be chosen between the 294 migration time of the neutral marker and the one of the RNSOs. This 295 parameter was optimized for GSNO and CysNO. CysNO, another important 296 nitrosothiol, as it is smaller than GSNO and with similar charge at this $\mathrm{pH}$, 297 presents a higher apparent mobility than GSNO. The best signal intensities 298 were obtained for an inversion time of $90 \mathrm{~s}$ and $75 \mathrm{~s}$, for GSNO and CysNO 299 respectively (Figure 5). These results indicate the versatility of the 
300 procedure for all types of RSNO. In these experimental conditions, the 301 analytical performances of this methodology were determined for GSNO. 302 The linearity was verified in the $100-700 \mu \mathrm{M}$ concentration range $(y=0.0485$ $\left.303 x-5.0485, R^{2}=0.9936\right)$ with a LOD of $20 \mu \mathrm{M}$

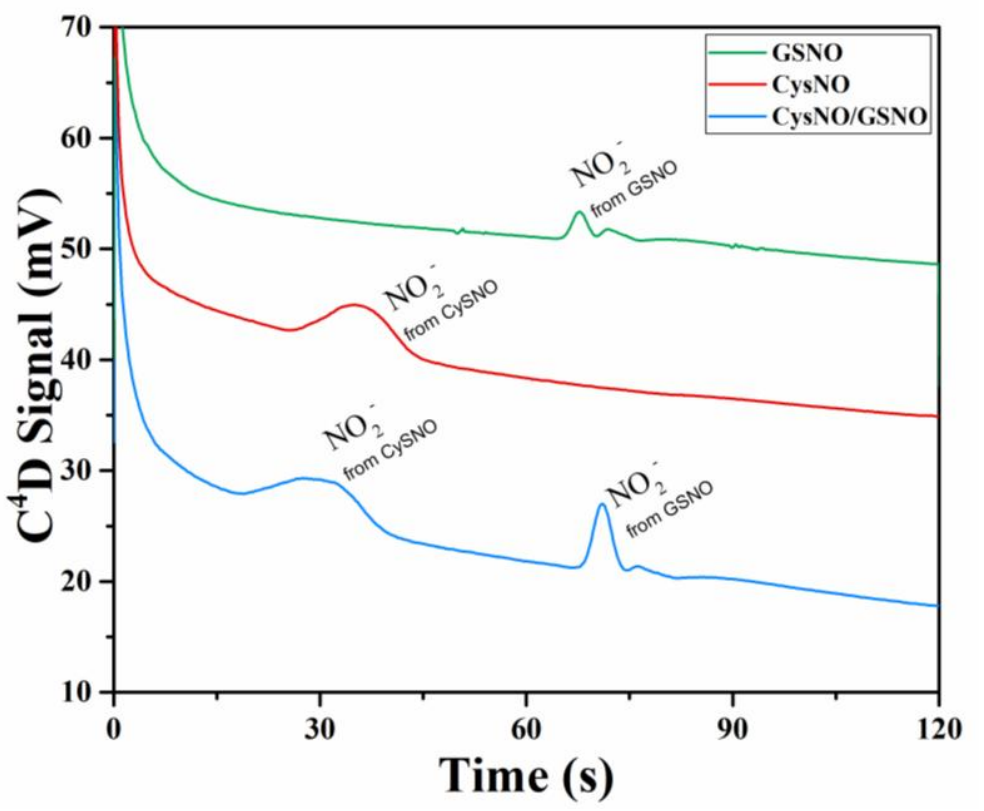

Figure 5 - Electropherograms for detection of $1 \mathrm{mM}$ GSNO (Black) and $1 \mathrm{mM}$ CySNO (Red and Blue) to determine the time of polarity inversion. In black and red $90 \mathrm{~s}$ was used for inversion while in blue $75 \mathrm{~s}$. BGE: $20 \mathrm{mM}$ arginine solution adjusted at pH 5.6 with acetic acid.

The final objective of such a micro-total analysis system is to allow for the simultaneous quantitation of various RSNOs. Therefore three main 311 challenges have to be adressed : (i) the efficient separation of the different 312 RSNOs, (ii) the choice of a unique time of polarity inversion in the process, 313 and (iii) the efficient detection of the sucessive nitrite zones produced from 314 each RSNOs decomposition. 
315 The simultaneous separation of GSNO and CysNO was performed so as to 316 prove the versatility of the sytem. For this purpose, GSNO and CysNO were

317 first separated and detected individually. The crucial parameter being the 318 inversion time, different values were applied from 75 to $90 \mathrm{~s}$. In a second 319 step, an equimolar mixture of CySNO and GSNO (1 mM each) was 320 separated and detected. Figure 6 presents the resulting electropherograms 321 for an optimized inversion time of $75 \mathrm{~s}$ that corresponds to the migration 322 time of CysNO. As expected the signal arising from GSNO appears after 323 the one of CysNO, as CysNO has a higher apparent mobility. These results 324 indicate a powerfull simultaneous separation and indirect detection of 325 GSNO and CysNO. The similar intensities for GSNO and CySNO, injected 326 at the same concentration indicate that decomposition efficiency is similar 327 in both cases. This method is therefore applicable for the quantitation of 328 pharmaceutical RSNOs, future drug candidates. Some work is in progress 329 for decreasing the LODs to reach biological concentrations (less than 16 $\left.330 \mu \mathrm{M}^{22}\right)$. 


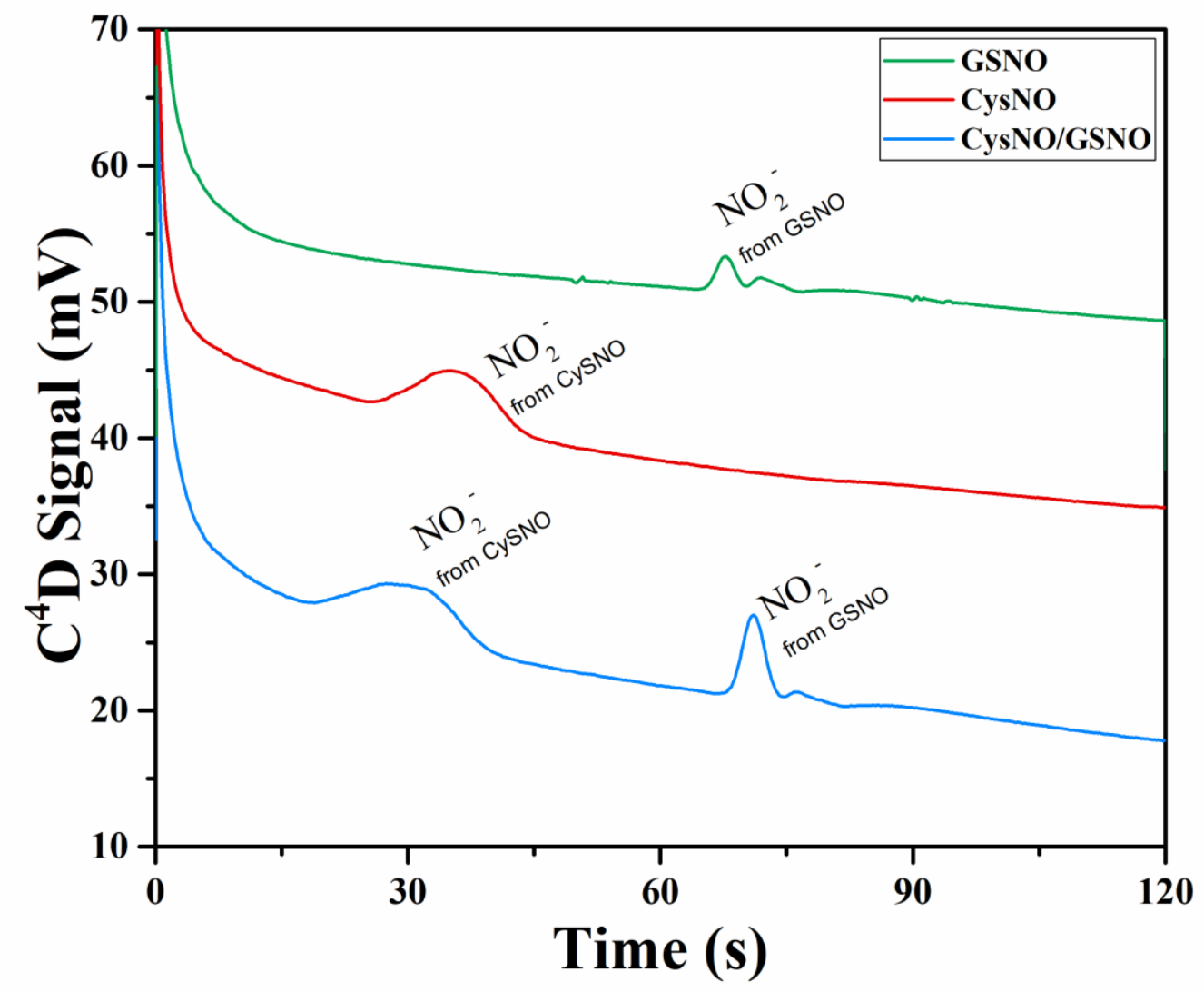

333 Figure 6 - Electropherograms corresponding to the electrophoretic profile of CySNO (1 mM), GSNO (1 $\mathrm{mM})$ and mixture GSNO (1 $\mathrm{mM})$ / CySNO $(1 \mathrm{mM})$ in blue, red and black, respectively. Experiments were performed in Su-8/pyrex microchip with gated injection. Procedure : (1) 3s injection, (12) application of $\mathrm{V} 1=800 \mathrm{~V}, \mathrm{~V} 2=1000 \mathrm{~V}$, during 3 minutes (not visible on the graph as it occurs before running the electropherogram), (3) addition of $\mathrm{Hg}^{2+}$ at $\mathrm{t}=0 \mathrm{~s}$ of the electropherogram, and inversion of polarity $\mathrm{V} 1=-$ $800 \mathrm{~V}, \mathrm{~V} 2=-1000 \mathrm{~V}$ for $\mathrm{t}=100 \mathrm{~s}$. Detection 1V vs Pt. BGE: ARG $20 \mathrm{mM}$ adjusted to $\mathrm{pH} 5.8$ with HAc 


\section{Conclusions}

340 An original method to simultaneously quantify two low molecular RSNOs in

341 a mixture using MCE was developed. A commercial microchip of SU-

342 8/Pyrex microchip and a wireless isolated potentiostat were used. After the

343 electrokinetic separation step, an inversion of electrokinetic polarity was

344 necessary to mix the RSNOs with the decomposition agent within the

345 separation channel and detect the produced nitrite by amperometric

346 detection at the buffer waste reservoir. Optimization of BGE composition

347 and detection potential were performed in order to obtain the best signal

348 intensity and $\mathrm{S} / \mathrm{N}$ ratio. The LODs were $20 \mu \mathrm{M}$ for GSNO and CysNO. This

349 methodology can be applied for the quantitation of pharmaceutical RSNOs,

350 future drug candidates. Using a more environmental friendly decomposition

351 reagent such as immobilized gold nanoparticles is envisaged. The method

352 developed herein has shown to be versatile, opening the way to the

353 quantitation of complex mixtures of RSNOs.

354

355 Conflicts of interest

356 The author declares no conflicts of interest.

357 Acknowledgements 
Financial support from "Coordenação de Aperfeiçoamento de Pessoal de

359

360

361

362

363

364

365

366

367

368

369

370

371

372

373

374

375

376

377

378

379

380

381

382

383

384

385

386

387

388

389

390

391

392

393

394

395

396

Nível Superior (CAPES)" and "French Committee for the Evaluation of

Academic and Scientific Cooperation with Brazil (COFECUB)" (grant $n^{\circ}$

802-14) is acknowledged.

\section{References}

1. B. T. Mellion, L. J. Ignarro, C. B. Myers, E. H. Ohlstein, B. A. Ballot, A. L. Hyman and P. J. Kadowitz, Mol. Pharmacol., 1983, 23, 653-664.

2. J. S. Stamler, D. I. Simon, J. A. Osborne, M. E. Mullins, O. Jaraki, T. Michel, D. J. Singel and J. Loscalzo, Proc. Natl. Acad. Sci. U.S.A., 1992, 89, 444-448.

3. E. J. Langford, A. S. Brown, R. J. Wainwright, A. J. Debelder, M. R. Thomas, R. E. A. Smith, M. W. Radomski, J. F. Martin and S. Moncada, Lancet, 1994, 344, 1458-1460.

4. M. W. Radomski, D. D. Rees, A. Dutra and S. Moncada, Br. J. Pharmacol., 1992, 107, 745-749.

5. G. F. P. de Souza, J. K. U. Yokoyama-Yasunaka, A. B. Seabra, D. C. Miguel, M. G. de Oliveira and S. R. B. Uliana, Nitric Oxide-Biol. Chem., 2006, 15, 209-216.

6. C. G. Kevil and R. P. Patel, Curr. Opin. Investig. Drugs, 2010, 11, 1127-1134.

7. M. Ito, Annu. Rev. Neurosci., 1989, 12, 85-102.

8. A. R. Butler and P. Rhodes, Anal. Biochem., 1997, 249, 1-9.

9. K. M. Beeh, J. Beier, N. Koppenhoefer and R. Buhl, Chest, 2004, 126, 11161122.

10. V. A. Tyurin, S. X. Liu, Y. Y. Tyurina, N. B. Sussman, C. A. Hubel, J. M. Roberts, R. Taylor and V. E. Kagan, Circ. Res., 2001, 88, 1210-1215.

11. A. B. Milsom, C. J. H. Jones, J. Goodfellow, M. P. Frenneaux, J. R. Peters and P. E. James, Diabetologia, 2002, 45, 1515-1522.

12. M. W. Foster, T. J. McMahon and J. S. Stamler, Trends Mol. Med., 2003, 9, 160.

13. B. C. Smith and M. A. Marletta, Curr. Opin. Chem. Biol., 2012, 16, 498-506.

14. A. Ismail, F. d'Orlyé, S. Griveau, J. A. F. Da Silva, F. Bedioui and A. Varenne, Anal. bioanal. chem., 2015, 407, 6221-6226.

15. A. Ismail, F. d'Orlye, S. Griveau, F. Bedioui, A. Varenne and J. A. F. da Silva, Electrophoresis, 2015, 36, 1982-1988.

16. S. Griveau and F. Bedioui, Analyst, 2013, 138, 5173-5181.

17. D. Giustarini, A. Milzani, I. Dalle-Donne and R. Rossi, J. Chromatogr. B, 2007, 851, 124-139.

18. D. L. H. Williams, Acc. Chem. Res., 1999, 32, 869-876.

19. M. M. Veleeparampil, U. K. Aravind and C. T. Aravindakumar, adv. phys. chem., 2009, 2009.

20. M. G. de Oliveira, S. M. Shishido, A. B. Seabra and N. H. Morgon, J. Phys. Chem. A, 2002, 106, 8963-8970. 
21. H. R. Swift and D. L. H. Williams, J. Chem. Soc., Perkin Trans. 2, 1997, 19331935.

22. A. Ismail, M. O. Araujo, C. L. S. Chagas, S. Griveau, F. D'Orlye, A. Varenne, F. Bedioui and W. K. T. Coltro, Analyst, 2016, 141, 6314-6320.

23. R. A. Hunter, B. J. Privett, W. H. Henley, E. R. Breed, Z. Liang, R. Mittal, B. P. Yoseph, J. E. McDunn, E. M. Burd, C. M. Coopersmith, J. M. Ramsey and M. H. Schoenfisch, Anal. Chem., 2013, 85, 6066-6072.

24. R. A. Hunter and M. H. Schoenfisch, Anal. Chem., 2015, 87, 3171-3176.

25. D. B. Gunasekara, M. K. Hulvey, S. M. Lunte and J. A. F. da Silva, Anal. Bioanal. Chem., 2012, 403, 2377-2384.

26. F. Q. Tu, L. Y. Zhang, X. F. Guo, Z. X. Zhang, H. Wang and H. S. Zhang, J. Chromatogr. A, 2014, 1359, 309-316.

27. J.-W. Yoo, G. Acharya and C. H. Lee, Biomaterials, 2009, 30, 3978-3985.

28. L. A. Peterson, T. Wagener, H. Sies and W. Stahl, Chem. Res. Toxicol., 2007, 20, 721-723.

29. R. A. Hunter and M. H. Schoenfisch, Anal. Chem., 2015, 87, 3171-3176.

30. https://www.micruxfluidic.com/en/ Acessed on 04/12/2018

31. S. C. Jacobson, S. V. Ermakov and J. M. Ramsey, Anal. Chem., 1999, 71, 32733276.

32. A. Ismail, S. Griveau, F. d'Orlyé, A. Varenne and F. Bedioui, Electroanalysis, 2015, 27, 2857-2863.

33. B. Thirumalraj, S. Palanisamy, S.-M. Chen and D.-H. Zhao, J. Colloid Interface Sci., 2016, 478, 413-420.

34. P. Kubáň and P. C. Hauser, Electrophoresis, 2009, 30, 3305-3314.

35. J. L. Beckers and P. Boček, Electrophoresis, 2003, 24, 518-535. 